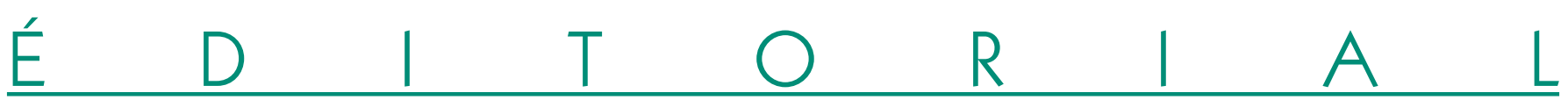

\title{
Le bon, la brute et l'A.T.M.
}

L'Articulation Temporo-Mandibulaire (A.T.M.) est une structure anatomique complexe et spécifique. Elle offre une grande capacité de mouvement à la mandibule. Mais en même temps, elle assure la stabilité condylienne quelle que soit la position mandibulaire, tout en maintenant un fort potentiel d'absorption viscoélastique des contraintes. Ceci est en grande partie lié à la structure et la forme du disque articulaire et à la cohésion condylo-discale (union). La souplesse de la structure fibro-cartilagineuse, la grande surface de la membrane synoviale, le fort volume liquidien, caractéristiques de cette articulation, lui donnent ses capacités de « résilience » ou réserve viscoélastique, de modelage et de remodelage et soulignent l'importance du mouvement dans les échanges trophiques.

\section{Aller de l'avant}

Pour ce qui est de la position "centrée ", la morphologie de la fosse mandibulaire du temporal, ouverte en arrière et latéralement, montre que l'ensemble condylo-discal ne peut trouver une zone de stabilisation qu'en avant contre le tubercule articulaire et un peu sur la zone médiale de la fosse mandibulaire. L'A.T.M. humaine est structurellement organisée pour être stabilisée vers l'avant par un ensemble musculaire maintenant la coaptation de l'ensemble condylo-discal contre le versant postérieur du tubercule.

\section{Adaptable mais fragile}

Quand on est jeune, on est adaptable mais aussi fragile; ce qui est vrai pour le psychisme l'est vraisemblablement pour l'Articulation Temporo-Mandibulaire. L'A.T.M., par son condyle, est jeune longtemps, beaucoup plus longtemps que toutes les autres articulations. Adaptable signifie tolérance, capacité de modelage en relation avec le contexte fonctionnel ou dysfonctionnel, mais, adaptable, implique aussi que l'expression symptomatologique est faible, que les alertes de surcharge sont rarement audibles. Fragile signifie moins résistante, il est possible que les processus désénératifs puissent se développer plus rapidement. 
A partir de ces principes, on peut décrire l'attitude de deux praticiens caricaturalement opposés:

\section{Le bon}

II sait que l'A.T.M de son jeune patient est fragile, mal stabilisée par les ligaments souvent lâches. II recherchera par un examen clinique attentif des signes «ténus » de désunion condylo-discale, car il pense que, si la plainte douloureuse ou le blocage sont rares, une simple désunion condylodiscale de grade l est probablement associée à une moindre capacité d'absorption des contraintes. Il évitera de créer, en cours de traitement, des rétropositions par une manipulation mandibulaire forcée, par la création de contacts occlusaux reculant la mandibule, ou bridant ses mouvements.

\section{La brute}

Elle pensera que le condyle doit être bien calé au fond de la fosse mandibulaire, qu'un claquement articulaire est d'une grande banalité n'ayant aucune incidence sur la capacité d'absorption des contraintes, que la douleur est le seul élément clinique à prendre en considération, que face à une plainte, il faut arrêter le traitement quelque temps pour le reprendre (le même) par la suite.

Elle pensera que le disque est inutile et pourra même aller jusqu'à dire que les A.T.M. ne servent à rien en donnant l'exemple du patient qui est encore capable d'ouvrir la bouche après une condylectomie bilatérale!

A l'heure actuelle, beaucoup plus que les faibles preuves scientifiques dont nous disposons, les simples observations anatomophysiologiques, en particulier l'anatomie comparée avec les autres espèces, doivent éclairer notre réflexion pour discerner les capacités de tolérance et les potentialités pathogènes. Selon l'adage Aristotélicien : «la nature ne fait rien en vain » : un disque articulaire bien en place est bénéfique. Lorsqu'il n'est pas en place, la tolérance est vraisemblablement diminuée donc l'exigence thérapeutique doit être renforcée. En cas de traitement orthodontique, indiqué pour des raisons dentaires, il faut alors favoriser le calage occlusal et la liberté des mouvements mandibulaires, éviter la rétroposition, le verrouillage et la surcharge parafonctionnelle. 